\title{
In vitro evaluation of heparinized Cuprophan hemodialysis membranes
}

\author{
W. L. J. Hinrichs, H. W. M. ten Hoopen, G. H. M. Engbers, and J. Feijen* \\ Institute for Biomedical Technology and Faculty of Chemical Technology, University of Twente, P.O. Box 217, \\ 7500 AE Enschede, The Netherlands
}

Cuprophan hemodialysis membranes can be heparinized using $N, N^{\prime}$-carbonyldiimidazole (CDI) as a coupling agent. In this study, the characteristics of heparinized Cuprophan membranes have been evaluated. After immobilization, heparin partially retained its biologic activity. An anticoagulant activity of $12.4 \pm 4.2 \mathrm{mU} / \mathrm{cm}^{2}$ was measured using a thrombin inactivation assay. Immobilized heparin also displayed an anti-complement activity. After contact with human serum, heparinized Cuprophan induced no generation of significant amounts of fluid phase terminal complement complex (TCC), whereas untreated Cuprophan induced the generation of substantial amounts of TCC. Heparinization did not affect the permeability of Cuprophan for model solutes with molecular weights up to $12,000 \mathrm{~g} / \mathrm{mol}$ except for sulfobromophthalein sodium salt. The permeability of Cupro- phan for sulfobromophthalein sodium salt was slightly decreased after heparinization. The ultrafiltration rate of $\mathrm{Cu}$ prophan increased by about 30\% after heparinization, probably owing to an increased swelling of the membrane in water. Heparinized Cuprophan incubated in phosphatebuffered saline at $37^{\circ} \mathrm{C}$ showed some release of heparin. These amounts of released heparin, however, were very low as compared to the amounts of heparin which are systemically administered during clinical hemodialysis treatment. It is concluded that Cuprophan membranes heparinized by means of the CDI-activation procedure are highly promising for application in hemodialyzers to be used for the treatment of patients with reduced or without systemic administration of heparin. (c) 1997 John Wiley \& Sons, Inc.

\section{INTRODUCTION}

In current clinical practice, membranes most frequently used for hemodialysis are based on cellulose. Advantages of this type of membrane over recently developed synthetic membranes such as polyacrylonitrile copolymer, poly(methyl methacrylate), and polysulfon are its relatively low cost, optimal water permeability, and extensive clinical experience with the material. ${ }^{1,2}$ The main disadvantage is its relatively poor blood compatibility, which is expressed by a rather strong activation of the coagulation and complement system and a drop in the leukocyte count. ${ }^{1,3-5}$

Heparin is administered to uremic patients treated with hemodialysis to avoid complications associated with blood-material interactions. ${ }^{2}$ A systemic administration of heparin, however, may cause undesired side effects such as internal hemorrhages and, eventually, osteoporoses. ${ }^{6,7}$

*To whom correspondence should be addressed.
An often-applied strategy to improve the blood compatibility of blood-contacting materials is to immobilize heparin onto their surfaces. ${ }^{1,7-9}$ Immobilized heparin may act as an anticoagulant for blood and an inhibitor of the complement system at the site where it is required while the disadvantages of systemic administration are avoided. The heparin-material linkage can be either physical, ionic, or covalent. ${ }^{1,7,9,10}$ With physical or ionic bonding, immobilized heparin may easily be released from the surface. ${ }^{7,9,11,12}$ For hemodialysis this release is undesired because a large amount of heparin may enter the blood, eventually leading to the same disadvantages as systemic heparinization. Furthermore, release of heparin may gradually reduce the blood compatibility of the membrane during hemodialysis. Covalent bonds are more stable. Many techniques have been developed to couple heparin covalently to biomaterials. ${ }^{1,7,9,11-13}$ Most of them are based on a two-step procedure whereby the biomaterial is first activated by a bi- or trifunctional agent, after which heparin is added to form the heparin-material linkage. ${ }^{14,15}$ The use of bior trifunctional agents may lead to crosslinking of the 
material. For hemodialysis membranes, crosslinking is unwanted because it may reduce the permeability of the membrane.

Engbers ${ }^{1}$ developed an alternative procedure for the heparinization of Cuprophan hemodialysis membranes. Heparin is first reacted with $N, N^{\prime}$-carbonyldiimidazole (CDI). Subsequently, the activated product is contacted with the membrane by which a covalent coupling between heparin and the membrane is established. In a previous study it was shown that heparinization of Cuprophan by means of the CDI-activation procedure does not affect the permeability of the membrane for urea, indicating that crosslinking of the membrane is avoided. ${ }^{16}$

In the present study, the properties of Cuprophan heparinized by means of the CDI-activation procedure are evaluated. The biologic activity of immobilized heparin is investigated. Furthermore, the effects of heparinization of the permeability of the membrane and the stability of the heparin-Cuprophan linkage are studied.

\section{MATERIALS AND METHODS}

\section{Materials}

Cuprophan $150 M$ was a gift of AKZO Nobel (Wuppertal, Germany). Formamide (synthesis grade; Merck, Darmstadt, Germany) was purified and dehydrated according to a method described by Verhoek et al. ${ }^{17}$ and stored beneath a blanket of dry nitrogen. Heparin from porcine intestinal mucosa (activity: 165 $\mathrm{U} / \mathrm{mg}$ ) was purchased from Diosynth (Oss, The Netherlands). Heparin also from procine intestinal mucosa of which $55-60 \%$ of the sodium ions was substituted by benzyltrimethylammonium ions (heparin-triton B) was purchased from HBG (Enschede, The Netherlands). Human antithrombin III (AT III; Sigma Chemical Company, St. Louis, MO) was purified using a heparin-Sepharose column ${ }^{18,19}$ (Sigma Chemical Company). Bovine albumin (Alb), creatinine, human thrombin, sulfobromophthalein sodium salt (SBP), and vitamin $B_{12}\left(\right.$ Vit $\left.B_{12}\right)$ were purchased from Sigma. Chromogenic substrate $S_{2238}$ was purchased from Chromogenix (Mölndal, Sweden). Polyethylene glycol $(6000 \mathrm{~g} / \mathrm{mol})$ (PEG) was purchased from Fluka Chemie (Buchs, Switzerland). PTT reagent was obtained from Boehringer (Mannhein, Germany). CPDA-1 plasma was purchased from the Rode Kruis Bloedbank Twente-Achterhoek (Enschede, The Netherlands). Phosphate-buffered saline (pH: 7.4) (PBS) was purchased from NPBI (Emmercompascuum, The Netherlands). CDI, cytochroom C, D (+) glucose, gla- cial acetic acid, glycerol, inulin, $\mathrm{NaCl}, \mathrm{Na}_{2} \mathrm{HPO}_{4}$, $\mathrm{NaH}_{2} \mathrm{PO}_{4}$, resorcinol, sucrose, toluidine blue $\mathrm{O}$ zinc chloride double salt, tris(hydroxymethyl)-ammonium methane (Tris), and urea (all of analytic grade) were purchased from Merck. A TCC ELISA was purchased from Quidel Corporation (Alkmaar, The Netherlands). All chemicals were used without further purification except when mentioned.

\section{Heparinization of Cuprophan}

Cuprophan was heparinized as described before (Fig. 1). ${ }^{1}$ Briefly, heparin-triton B was dissolved in dry formamide (heparin concentration: $0.20 \mathrm{~g} / \mathrm{mL}$ ) and subsequently activated with CDI (weight ratio heparin $/ C D I=10)$ at ambient temperature. After $1 \mathrm{~h}$, the reaction mixture was transferred to Cuprophan membranes, which were thoroughly prerinsed with formamide to remove glycerol and water. After 2 days of immobilization at ambient temperature, the membranes were thoroughly rinsed with demineralized water and then with aqueous $4 \mathrm{M} \mathrm{NaCl}$. Subsequently, the membranes were thoroughly rinsed with demineralized water, soaked in a $10 \mathrm{wt} \%$ solution of glycerol in demineralized water, and then dried by air exposure. Treatment of the membranes with an aqueous glycerol solution before drying is necessary to prevent an irreversible collapse of pores. ${ }^{20}$ Before the membranes were subjected to further testing, they were incubated in water or PBS at $4^{\circ} \mathrm{C}$ for $16 \mathrm{~h}$ to remove glycerol. The immobilization procedure yielded membranes with $60-80 \% \mu \mathrm{g}$ immobilized heparin $/ \mathrm{cm}^{2}$ membrane surface area as determined by means of a toluidine blue staining procedure as described by Smith et al. ${ }^{21}$ and modified for our purposes. ${ }^{22,23}$

\section{Anticoagulant activity of immobilized heparin}

The antithrombin activity of heparin immobilized onto Cuprophan was investigated by means of a thrombin inactivation assay developed by Chandler et al. ${ }^{24}$ This assay is based on two competitive reactions. The first reaction is heparin-catalyzed inactivation of thrombin by AT III. The seond reaction is thrombincatalyzed splitting of the chromogenic substrate $S_{2238}$ into a peptide and $p$-nitroaniline. The extinction of the latter yellow compound can be measured spectrophotometrically.

A buffer consisting of an aqueous solution of 50 $\mathrm{mmol} / \mathrm{L}$ Tris, $1.0 \mathrm{~g} / \mathrm{L}$ PEG, $1.0 \mathrm{~g} / \mathrm{L}$ BSA, and 150 mmol $\mathrm{NaCl}$ adjusted to $\mathrm{pH} 8.4$ was prepared. The buffer was used for the preparation of a substrate $S_{2238}$ 

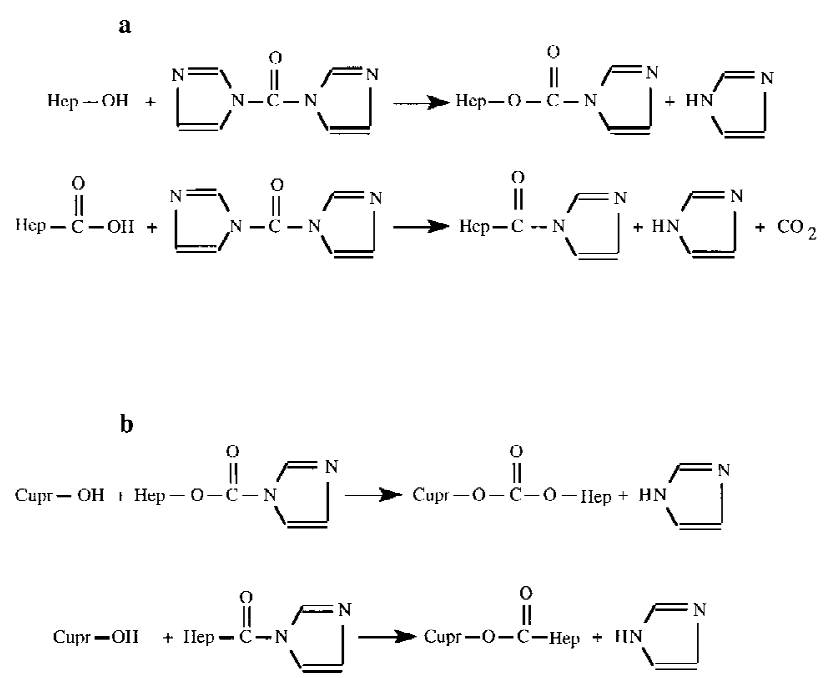

Figure 1. Heparinization of Cuprophan by means of a CDI activation procedure. (a) Activation reactions of heparin with CDI. (b) Immobilization reactions of CDI-activated heparin onto Cuprophan.

$(2.0 \mathrm{mg} / \mathrm{mL}) /$ AT III $(70 \mathrm{mU} / \mathrm{ml})$ solution, a thrombin solution $(0.4 \mathrm{U} / \mathrm{mL})$, and a series of solutions of various heparin concentrations $(0-0.4 \mathrm{U} / \mathrm{mL})$. Membranes were cut into discs with a diameter of $5 \mathrm{~mm}$ and placed into wells of a 96-well plate containing $150 \mu \mathrm{L}$ of substrate/AT III and $40 \mu \mathrm{L}$ of buffer solution. For the determination of a calibration curve, $150 \mu \mathrm{L}$ of substrate/AT III solution and $40 \mu \mathrm{L}$ of heparin solutions were added to other wells. The reactions were initiated by adding $50 \mu \mathrm{L}$ of thrombin solution to the wells after which the well plate was shaken. After 10 $\mathrm{min}$, the reactions were quenched by adding $70 \mu \mathrm{L} 40$ vol \% acetic acid. Subsequently, $200 \mu \mathrm{L}$ of the resultant mixture was transferred into empty wells and the extinction was measured at $405 \mathrm{~nm}$. The reactions were carried out at room temperature. The results of experiments using solutions of known heparin concentrations were used to determine the activity of heparin immobilized onto Cuprophan. The experiments were performed at least four times.

\section{Anticomplement activity of immobilized heparin}

Blood was drawn from healthy volunteers into polypropylene tubes and allowed to clot for $1 \mathrm{~h}$ at room temperature. Subsequently, the samples were centrifuged at $2000 \times g$ for $15 \mathrm{~min}$ at $4^{\circ} \mathrm{C}$, after which the serum was separated from the clot by decantation. The serum was quickly frozen using liquid nitrogen and then placed at $-20^{\circ} \mathrm{C}$ until use.

Prior to the complement activation assay, serum was thawed at $37^{\circ} \mathrm{C}$ and stored on ice. Membranes were cut into discs with a diameter of $5 \mathrm{~mm}$. Ten of these membranes were placed in a polystyrene tube. Subsequently, $500 \mu \mathrm{L}$ of serum was added to the tube. Polystyrene tubes which did not contain membranes were used as negative controls. Thereafter, the tubes were gently shaken at $37^{\circ} \mathrm{C}$. At different time intervals samples of the serum were taken. The TCC concentrations of the samples were determined by means of an ELISA according to the recommendations of the manufacturer. The experiments were performed four times.

\section{Effect of heparinization on the permeability of Cuprophan}

The effect of heparinization on the permeability of Cuprophan for solutes of different molecular weights (Table I) was investigated using a Minitan-S ultrafiltration system (Millipore, Etten-Leur, The Netherlands) equipped with a peristaltic pump (WatsonMarlow Ltd., Cornwall, UK) or using a minibatch dialyzer (only for testing the permeability for cytochrome C). The experiments were carried out at $37^{\circ} \mathrm{C}$. In all cases, except for $\mathrm{PO}_{4}{ }^{3-}$, solutions of the solutes in PBS were used as feed and PBS as dialysate. In case of $\mathrm{PO}_{4}{ }^{3-}$, solutions of $\mathrm{NaH}_{2} \mathrm{PO}_{4}$ and $\mathrm{Na}_{2} \mathrm{HPO}_{4}$ in aqueous 0.42 wt \% NaCl were used as feed and aqueous 0.42 wt $\% \mathrm{NaCl}$ as dialysate. Solutions containing a mixture of urea, creatinine, and glucose and solutions containing a mixture of SBP and Vit $\mathrm{B}_{12}$ were also used. Preliminary experiments showed that the permeability of the membranes for one of these solutes was not affected by the presence of the other solute(s) in the same solution (data not shown). The starting concentrations of the solutes of the various feed solutions are listed in Table I. Samples were taken from the dialysate at different time intervals (except for $\mathrm{PO}_{4}{ }^{3-}$ ). The samples were analyzed as follows. Glucose: The concentration was determined using a Cobas Bio automatic analyzer and a glucose kinetic ultra violet (UV) test kit. Urea and creatinine: the concentrations were determined using a Hitachi 717 automatic analyzer and kinetic UV test kits for urea and creatinine. Sucrose and inulin ${ }^{25}: 1 \mathrm{~mL}$ of the sample was added to $5 \mathrm{~mL}$ of a $0.167 \mathrm{~g} / \mathrm{mL}$ solution of resorcinol in aqueous $4 \mathrm{~N} \mathrm{HCl}$. The resultant mixture was heated to $100^{\circ} \mathrm{C}$ for $15 \mathrm{~min}$. After cooling to ambient temperature, the extinction was measured at $480 \mathrm{~nm}$. Vit $\mathrm{B}_{12}$ : the extinction was measured at $360 \mathrm{~nm}$. SBP: the sample was $1 / 1(\mathrm{v} / \mathrm{v})$ diluted with aqueous $0.01 \mathrm{~N} \mathrm{NaOH}$, after which the extinction of the mixture was measured at $578 \mathrm{~nm} . \mathrm{PO}_{4}{ }^{3-}$ : the conductivity of the dialysate was measured in situ by means of a Philips PW 9527 conductivity flow cell. Cytochrome C: the extinction was 
measured at $409 \mathrm{~nm}$. Calibration curves were used to determine the concentrations of the solutes.

The mass transfer coefficient, $K_{o}$, was calculated using the formula as derived by Smith et al. ${ }^{26}$ :

$$
-\operatorname{Ln}\left(\Delta C_{t} / \Delta C_{o}\right)=K_{o} \times A \times t \times\left(1 / V_{1}+1 / V_{2}\right),
$$

where $\Delta C_{t}$ and $\Delta C_{o}$ are the concentration differences of the solutes over the membrane at times $t$ and 0 , respectively; $A$ is the membrane surface area; $t$ is the dialysis time; and $V_{1}$ and $V_{2}$ are the volumes of feed and dialysate respectively. All experiments were performed at least four times.

\section{Effect of heparinization on the ultrafiltration characteristics of Cuprophan}

The water permeability of nonheparinized and heparinized Cuprophan was determined with an Amicon 8050 ultrafiltration cell (Amicon, Capelle aan de Ijssel, The Netherlands). The membrane was positioned in the ultrafiltration cell, after which the cell was filled with deionized water. Subsequently, a pressure of 3 bar was applied. After 1-2 $\mathrm{h}$ of equilibration, the water flow through the membrane was measured for $2-4 \mathrm{~h}$. The measurements were performed in triplicate.

\section{Stability of heparin-Cuprophan linkage in PBS}

Heparinized Cuprophan was placed in tubes containing PBS which was supplemented with $\mathrm{NaN}_{3}$ (final concentration: $0.02 \mathrm{wt} \%$ ) to prevent bacterial growth. The tubes were placed in a water bath of $37^{\circ} \mathrm{C}$ and gently shaken. At different time intervals, samples of PBS were taken. The amount of heparin released into PBS was determined by means of an activated partial thromboplastin time (APTT) assay.

TABLE I

Molecular Weights and Starting Concentrations in the Feed of Solutes Used to Test the Permeability of the Membranes

\begin{tabular}{lcc}
\hline \multicolumn{1}{c}{ Solute } & $\begin{array}{c}\text { Molecular Weight } \\
(\mathrm{g} / \mathrm{mol})\end{array}$ & $\begin{array}{c}\text { Starting Concentration } \\
\text { in Feed (wt \%) }\end{array}$ \\
\hline Urea & 60 & 0.76 \\
Phosphate & 96 & $*$ \\
Creatinine & 113 & 0.035 \\
Glucose & 180 & 3.45 \\
Sucrose & 342 & 0.2 \\
SBP & 835 & 0.02 \\
Vitamin $\mathrm{B}_{12}$ & 1355 & 0.02 \\
Inulin & 5200 & 0.2 \\
Cytochrome $\mathrm{C}$ & 12,000 & 1.0 \\
\hline${ }^{*}$ Mixture of $\mathrm{Na}_{2} \mathrm{HPO}_{4}(0.9$ wt \%) & and $\mathrm{NaH}_{2} \mathrm{PO}_{4}(0.15$ \\
wt \%). &
\end{tabular}

Therefore, fresh-frozen human CPDA- 1 plasma was thawed at $37^{\circ} \mathrm{C}$ and stored on ice. To $50 \mu \mathrm{L}$ of the release medium, $50 \mu \mathrm{L}$ plasma was added and thoroughly mixed at $37^{\circ} \mathrm{C}$. After $1 \mathrm{~min}, 50 \mu \mathrm{g}$ PTT reagent was added. The resulting suspension was mixed for 3 $\min$ at $37^{\circ} \mathrm{C}$. Thereafter, $50 \mu \mathrm{L}$ of an aqueous $20 \mathrm{mM}$ $\mathrm{CaCl}_{2}$ solution was added. The suspension was mixed again and the coagulation time was determined with a coagulatometer (LC-6; Lode, Groningen, The Netherlands). A calibration curve, obtained using solutions of heparin in PBS of known activities, was applied to calculate the release of heparin from the membrane. The measurements were performed eight times.

\section{RESULTS AND DISCUSSION}

With the CDI-activation procedure, $60-80 \mu \mathrm{g}$ hepa$\mathrm{rin} / \mathrm{cm}^{2}$ membrane surface was immobilized. Baumann et al., ${ }^{11}$ Schmitt et al., ${ }^{12}$ and Holland et al., ${ }^{27}$ who studied the ionic binding of heparin onto cationized cellulosic membranes $\left[N, N^{\prime}\right.$-diethylaminoethyl cellulose and 2-(hydroxy-3-trimethylammoniumchloride) propyl cellulose] found comparable amounts of immobilized heparin. These very high amounts of immobilized heparin are remarkable. If a monolayer of heparin would have formed on the surface of the membranes, a density of only $0.1-0.2 \mu \mathrm{g} / \mathrm{cm}^{2}$ or 2.0 $\mu \mathrm{g} / \mathrm{cm}^{2}$ is estimated for heparin immobilized with a side-on or an end-on orientation, respectively. ${ }^{28}$ Water swollen Cuprophan has pores with a diameter up to $25 \AA .{ }^{29}$ Most likely, heparin is also immobilized in the pores of the membrane.

The anticoagulant activity of immobilized heparin was evaluated by measuring the inactivation of thrombin in a chromogenic assay. In this assay, heparinized Cuprophan displayed an anticoagulant activity of $12.4 \pm 4.2 \mathrm{mU} / \mathrm{cm}^{2}$, whereas untreated Cuprophan showed no significant activity $(0.5 \pm 0.6 \mathrm{mU} /$ $\mathrm{cm}^{2}$ ). These results show that heparin immobilized by means of the CDI-activation procedure at least partially retained its original anticoagulant activity. As mentioned above, heparin was partially immobilized in the pores of the membrane. It is expected that this part of immobilized heparin did not contribute to the anticoagulant activity because the pores are too small to allow for the penetration of AT III and thrombin. Therefore, only heparin immobilized at the surface will display anticoagulant activity. Depending on its orientation, a maximal activity of $16.5-350 \mathrm{mU} / \mathrm{cm}^{2}$ is calculated for a monolayer of immobilized heparin. This maximal activity was not reached for heparinized Cuprophan. Two reasons may account for this. First, a monolayer of immobilized heparin at the surface may not have formed. Second, the activity of heparin was reduced owing to immobilization. A reduction of the activity is usually observed upon immobilization, ${ }^{10,30}$ 
which can be ascribed to a restricted mobility of surface-bound heparin as compared to unbound heparin. As a consequence, the exposure of binding sites of heparin for AT III and thrombin is limited.

Complement activation induced by the membranes was determined by measuring the concentration of fluid phase TCC. As shown in Figure 2, untreated Cuprophan induced the generation of substantial amounts of fluid-phase TCC, which strongly increased during $3 \mathrm{~h}$ of incubation. The generation of fluidphase TCC induced by heparinized Cuprophan was much lower. The concentration of TCC in serum in the presence of heparinized Cuprophan also increased in time but did not significantly differ from the TCC concentration induced by the test tube (polystyrene) alone.

Reduced complement activation by the heparinized membranes can be ascribed to two different mechanisms. First, it is generally assumed that the hydroxyl groups of cellulose trigger the alternative pathway of the complement system by covalently binding of $\mathrm{C} 3 \mathrm{~b} .{ }^{31,32}$ Therefore, masking or modifying of the hydroxyl groups of cellulose will reduce complement activation, which indeed has been observed by many investigators. ${ }^{31-36}$ Heparinization will also mask and modify the hydroxyl groups of Cuprophan, thereby passively reducing complement activation. Second, surface-immobilized heparin may also play an active role in reducing complement activation. As shown by Kazatchkine et al., ${ }^{37}$ immobilized heparin prevents the formation of the alternative pathway $\mathrm{C} 3$ amplification convertase, $\mathrm{C} 3 \mathrm{bBb}$, by promoting the interaction of the inhibiting proteins $\mathrm{H}$ and I with surface-bound $\mathrm{C} 3 \mathrm{~b}$.

Fluid-phase concentration of TCC is considered to be a good measure for complement activation. ${ }^{38-40} \mathrm{In}$ a previous study, we have shown that heparinization

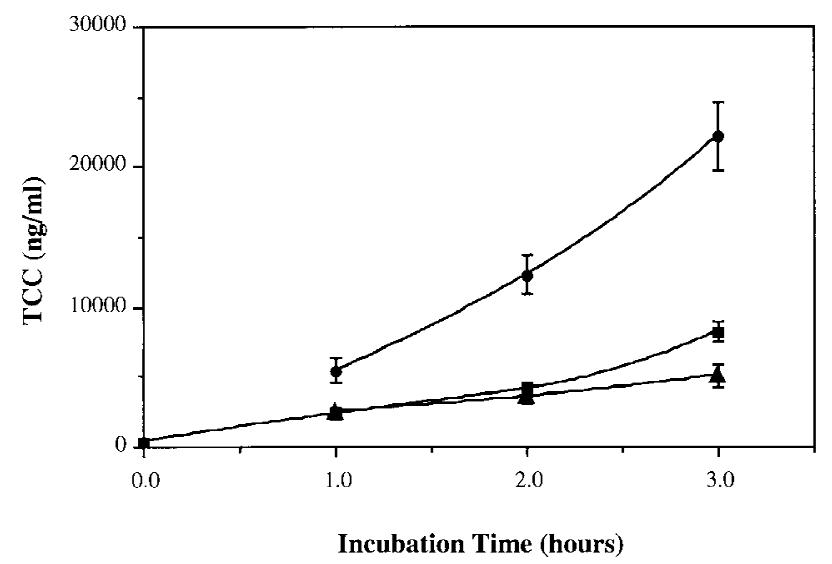

Figure 2. Generation of fluid-phase TCC induced by: untreated Cuprophan: - -; heparinized Cuprophan: $\mathbf{A}$; test tube (polystyrene) alone: - - Membranes with a surface area of $3.9 \mathrm{~cm}$ were added to $500 \mu \mathrm{L}$ of serum at $37^{\circ} \mathrm{C}$. The concentration of TCC in serum was measured by means of an ELISA $(n=3, \pm \mathrm{SD})$. of Cuprophan by means of the CDI-activation procedure strongly reduced the generation of fluid phase C3a desarg. ${ }^{16}$ Most frequently, fluid phase C3a desarg and C5a desarg concentrations are measured to quantify the degree of complement activation. However, care must be exercised to interpret the results of these measurements, because biomaterials can absorb these anaphylatoxins to different degrees depending on the surface characteristics of the materials under investigation. ${ }^{4,41-43}$ It has even been reported that in certain cases immobilized heparin has a high affinity for binding C3a. ${ }^{41,44}$ Deppisch et al. ${ }^{39}$ showed that TCC does not readily adsorb onto biomaterials. For heparinized materials, however, adsorption of TCC has not been investigated in detail, although Pekna et al., ${ }^{45}$ who studied complement activation by poly(methyl methacrylate) and heparinized poly(methyl methacrylate), showed that total C3 activation and fluid phase TCC generation correlated well. Nevertheless, it is possible that based on the results presented in this study, the anticomplement activity of heparin immobilized onto Cuprophan is overestimated. Adsorption of complement factors onto the membranes will therefore be the subject of future investigations.

Heparinization does not significantly affect the mass transfer coefficients for model solutes with molecular weights up to 12,000, except for SBP. The permeability of Cuprophan for SBP was slightly decreased after heparinization (Table II). The reduction of the permeability for SBP by heparinization is probably due to the negative charges of both SBP and heparin at physiologic conditions. Electrostatic repulsion will limit the diffusion of negatively charged SBP through the membrane onto which negatively charged heparin is immobilized.

Ultrafiltration experiments showed that heparinization increased the water permeability from $300 \pm 4$ $\mu \mathrm{L} / \mathrm{h} \times \mathrm{cm}^{2}$ to $385 \pm 11 \mu \mathrm{L} / \mathrm{h} \times \mathrm{cm}^{2}$ at a pressure of 3 bar. The increase is most likely because heparin is immobilized in the pores of the membranes, which results in an increase of the swelling of the membrane in

TABLE II

Mass Transfer Coefficients of Various Solutes for Untreated and Heparinized Cuprophan $(n \geqslant 4, \pm \mathrm{SD})$

\begin{tabular}{lcc}
\hline \multirow{2}{c}{ Solute } & \multicolumn{2}{c}{$K_{o}\left(10^{-3} \mathrm{~cm} / \mathrm{min}\right)$} \\
\cline { 2 - 3 } \multicolumn{1}{c}{$\begin{array}{c}\text { Untreated } \\
\text { Cuprophan }\end{array}$} & $\begin{array}{c}\text { Heparinized } \\
\text { Cuprophan }\end{array}$ \\
\hline Urea & $17.4 \pm 0.9$ & $17.9 \pm 1.5$ \\
Phosphate & $17.8 \pm 1.5$ & $18.3 \pm 1.6$ \\
Creatinine & $11.0 \pm 0.6$ & $12.1 \pm 1.1$ \\
Glucose & $7.36 \pm 0.42$ & $8.63 \pm 0.89$ \\
Sucrose & $5.06 \pm 0.39$ & $4.94 \pm 0.33$ \\
SBP & $3.93 \pm 0.25$ & $2.90 \pm 0.21$ \\
Vitamin $B_{12}$ & $1.73 \pm 0.09$ & $1.78 \pm 0.16$ \\
Inulin & $0.51 \pm 0.03$ & $0.42 \pm 0.07$ \\
Cytochrome C & $0.021 \pm 0.002$ & $0.026 \pm 0.005$ \\
\hline
\end{tabular}


water. A higher water permeability may require slight adjustments of the conditions during hemodialysis. These adjustments, however, are only very moderate compared to those required when synthetic membranes (e.g., polyacrylonitrile copolymer, polysulfone) are used. Synthetic membranes exhibit an ultrafiltration rate more than 10 times higher than that of cellulosic membranes ${ }^{5,46,47}$ and require special precautions to prevent excessive ultrafiltration during hemodialysis. ${ }^{5}$

Crosslinking of the membrane will result in a decreased permeability. In a control experiment a solution of CDI in formamide was added to Cuprophan. By this treatment, the membrane became very brittle, indicating crosslinking (data not shown). Crosslinking is likely to occur by CDI activation of part of the hydroxyl groups of Cuprophan followed by the formation of carbonate crosslinks, which results from the reaction between the activated hydroxyl groups and other hydroxyl groups of Cuprophan. ${ }^{48}$ During the heparinization procedure, heparin is activated with CDI in the absence of Cuprophan until all CDI has been consumed. ${ }^{22}$ Therefore, CDI is not contacted with the membrane and the crosslinking reaction as described above will not occur. This is confirmed by the results of the permeability experiments. It is likely, however, that more than one CDI-activated group per heparin molecule had reacted with the hydroxyl groups of Cuprophan, thus forming crosslinks. Apparently, these type of crosslinks do not affect the permeability of the membrane.

The stability of the heparin-Cuprophan linkage was studied by incubating heparinized Cuprophan in PBS at $37^{\circ} \mathrm{C}$ up to $6 \mathrm{~h}$. The release of heparin from the membrane was evident (Fig. 3). During activation of heparin with CDI, carboxylic acid and/or hydroxyl groups of heparin react with CDI to form imidazolyl amide and imidazolyl carbamate groups, respectively. ${ }^{48}$ After contact with Cuprophan, these activated groups react with the hydroxyl groups of the membrane to form ester and/or carbonate bonds, respectively. ${ }^{48}$ The carbonyl groups of these bonds give rise to an absorption at $1740 \mathrm{~cm}^{-1}$. FTIR spectroscopy revealed that at prolonged incubation times of heparinized Cuprophan in PBS at $37^{\circ} \mathrm{C}$ (1 week), the intensity of the absorption at $1740 \mathrm{~cm}^{-1}$ clearly reduces, indicating that the release of heparin was caused by the hydrolysis of covalent bonds between heparin and Cuprophan and not by the diffusion of physically bound heparin from the membrane (data not shown).

The release of heparin from the membrane plotted as a fuction of the incubation time yields a straight line (Fig. 3). This line does not cross the origin. Apparently, there is a burst effect in the release of heparin during the first 1 or $2 \mathrm{~h}$ of incubation. The following mechanism can be proposed to explain this behavior. Prior to the release experiment, the membranes were incubated in PBS at $4^{\circ} \mathrm{C}$ for $16 \mathrm{~h}$. During that time, some

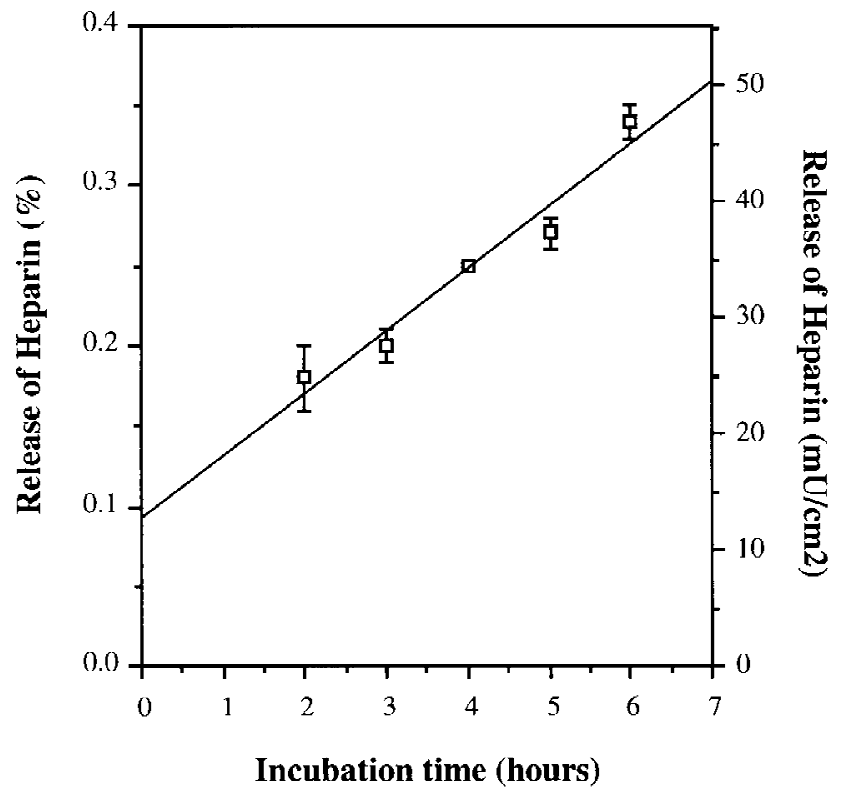

Figure 3. Release of heparin from heparinized Cuprophan incubated in PBS at $37^{\circ} \mathrm{C}$. The concentration of heparin in PBS was measured by means of an APPT assay $(n=8, \pm \mathrm{DS})$.

hydrolysis of the covalent bonds between heparin and Cuprophan will take place. Hydrolysis will result in a release of heparin from the membrane. However, part of the heparin which is no longer covalently linked may be physically entrapped in the pores of the membrane. When the membrane is transferred into PBS of $37^{\circ} \mathrm{C}$, the swelling of the membrane will increase. The increased swelling may facilitate the release of physcially entrapped heparin, resulting in a burst effect.

Assuming that the stability of the heparinCuprophan linkage is the same in blood as in PBS, the clinical relevance of the stability studies can be evaluated. Clinically applied dialyzers have a membrane surface area of $1.0-1.5 \mathrm{~m}^{2}$; the dialysis time is usually $4 \mathrm{~h}$. After $4 \mathrm{~h}$ of incubation in $\mathrm{PBS}$ at $37^{\circ} \mathrm{C}, 33.3 \pm 1.7$ $\mathrm{mU}$ of heparin $/ \mathrm{cm}^{2}$ was released from the membrane. This amount of released heparin seems very large compared with the activity of immobilized heparin as measured by means of the thrombin inactivation assay $\left(12.4 \pm 4.2 \mathrm{mU} / \mathrm{cm}^{2}\right)$. However, the activity of released heparin cannot be compared with the activity of the membrane. As mentioned, a large amount of heparin is immobilized in the pores of the membrane and does not contribute to the anticoagulant activity. As indicated above, the hydrolysis of covalent bonds between heparin and Cuprophan resulting in a release of heparin is not limited to the surface but also occurs in the pores of the membrane. Most likely, the relative amount of heparin released from the surface and from the pores of the membrane will be the same. Therefore, most of the released heparin had not contributed to the anticoagulant activity of the membrane. Furthermore, because immobilized heparin exhibits a 
lower activity than heparin in solution, the activity of the fraction of heparin which is released from the surface as measured in the release experiments will be higher than its activity when it is still immobilized and measured by means of the thrombin inactivation assay. In a previous study, ${ }^{22}$ heparin was activated with CDI after which the activated groups were hydrolyzed. It was shown that the activity of heparin was not affected by this treatment. Therefore, it can be assumed that heparin, before being used for heparinization, has the same activity as heparin released from the membrane. Thus, the amount of released heparin can be expressed as a percentage of the original amount of immobilized heparin. After $4 \mathrm{~h}$ of incubation in PBS at $37^{\circ} \mathrm{C}$, a release of $0.25 \%$ can be calculated. Because $99.75 \%$ of the original amount of heparin is still immobilized, a significant decrease of the blood compatibility is not expected.

After $4 \mathrm{~h}$ of incubation in $\mathrm{PBS}$ at $37^{\circ} \mathrm{C}, 33.3 \pm 1.7 \mathrm{mU}$ of heparin $/ \mathrm{cm}^{2}$ was released from the membrane. For a membrane surface area of $1.0-1.5 \mathrm{~m}^{2}$, this would mean that 330-500 $\mathrm{U}$ of heparin will be released from the membrane during $4 \mathrm{~h}$ of hemodialysis. A release of $330-500 \mathrm{U}$ of heparin is much less than $4000-15,000 \mathrm{U}$ of heparin, which is systemically administered during hemodialysis ${ }^{2}$ and is not expected to cause any undesired effects. The release of heparin may even be reduced when the burst effect as described above can be prevented. This may be achieved by rinsing the membrane with an aqueous solution at $37^{\circ} \mathrm{C}$ prior to hemodialysis, which is usually done in clinical practice.

\section{CONCLUSIONS}

The results of the present study clearly show that heparin immobilized onto Cuprophan hemodialysis membranes by means of the CDI-activation procedure retains part of its biologic activities as expressed in the thrombin inactivation and TCC assays. Furthermore, the heparinization procedure does not have an adverse effect on the permeability of the membrane. The release of heparin from the membrane upon incubation in PBS is very low compared to the amount of heparin systemically administered during hemodialysis. Therefore it can be concluded that Cuprophan membranes heparinized by means of the CDIactivation procedure are highly promising for application in hemodialyzers to be used for the treatment of patients with reduced, or without systemic administration of, heparin.

This study was financially supported by the Dutch Kidney Foundation (Grant C 91.1077). The authors thank J. A. Korf (MST, Enschede, The Netherlands) for analysis of the glucose, sucrose, and urea samples; and Dr. C. J. van Delden
(Institute for Biomedical Technology and Faculty of Chemical Technology, University of Twente, Enschede, The Netherlands), for assistance with the thrombin inactivation assay.

\section{References}

1. G. H. M. Engbers, "The development of heparinized materials with an improved blood compatibility," Thesis, University of Twente, Enschede, The Netherlands, 1990.

2. H. Ireland, P. Rylance, and P. Kesteven, "Heparin as an anticoagulant during extracorporal circulation," in Heparin. Chemical and Biological Properties. Clinical Applications, D. A. Lane and U. Lindahl(eds.), Edward Arnold, London, 1989, pp. 549-574.

3. D. Docci, C. Delvecchio, F. Turci, L. Baldrati, and C. Gollini, "Effect of different dialyzer membranes on serum angiotensin-converting enzyme during hemodialysis," Int. J. Artif. Organs, 11, 28-32 (1988).

4. A. K. Cheung, D. E. Chenoweth, D. Otsuka, and L. W. Henderson, "Compartmental distribution of complement activation products in artifical kidneys," Kidney Int., 30, 74-80 (1986).

5. A. v. d. Steen, "Research on dialyzers with improved biocompatibility," Clin. Nephrol., 26, S39-S42 (1986).

6. M. N. Levine, J. Hirsh, and J. G. Kelton, "Heparininduced bleeding," in Heparin. Chemical and Biological Properties. Clinical Applications, D. A. Lane and U. Lindahl (eds.), Edward Arnold, London, 1989, pp. 517-574.

7. O. Larm, R. Larsson, and P. Olsson, "Surfaceimmobilized heparin," in Heparin. Chemical and Biological Properties. Clinical Applications, D. A. Lane and U. Lindahl (eds.), Edward Arnold, Longon, 1989, pp. 597 608.

8. M. F. A. Goosen, M. V. Sefton, and M. W. C. Hatton, "Inactivation of thrombin by antithrombin III on a heparinized biomaterial," Thromb. Res., 20, 543-554 (1980).

9. G. H. M. Engbers and J. Feijen, "Current techniques to improve the blood compatibility of biomaterial surfaces," Int. J. Art. Org., 14, 199-215 (1991).

10. M. Amiji and K. Park, "Surface modification of polymeric biomaterials with poly(ethylene oxide), albumin, and heparin for reduced thrombogenicity," J. Biomater. Sci. Polym. Edn., 4, 217-234 (1993).

11. H. Baumann, R. Keller, and E. Ruzicka, "Partially cationized cellulose for non-thrombogenic membrane in the presence of heparin and endothelial-cell-surfaceheparansulfate (ES-HS)," J. Membr. Sci., 61, 253-268 (1991).

12. E. Schmitt, M. Holtz, H. Klinkmann, G. Esther, and J. M. Courtney, "Heparin binding and release properties of DEAE cellulose membranes," Biomaterials, 4, 309-313 (1983).

13. O. Larm, R. Larsson, and P. Olsson, "A new nonthrombogenic surface prepared by selective covalent binding of heparin via modified reducing terminal residue," Biomat. Med. Dev. Art. Org., 11, 161-173 (1983).

14. H. Hasenfratz and G. Knaup, "Improvement of the blood compatibility of cellulosic membranes through the immobilization of heparin and measurement of biological heparin activity," Artif. Organs, 5, 507-511 (1981).

15. G. Schmer, L. N. L. Teng, J. J. Cole, J. E. Vizzo, M. M. Francisco, and B. H. Scribner, "Successful use of a totally heparin grafted hemodialysis system in sheep," Trans. Am. Soc. Artif. Intern. Organs, 32, 654-662 (1976). 
16. G. H. M. Engbers, T. v. Mechelen, B. M. T. Croonen, L. Robertson, J. M. Courtney, and J. Feijen, "Heparinized cuprophan," Adv. Biomater., 9, 487-492 (1990).

17. F. H. Verhoek, "The strength of acids in formamide," $J$. Am. Chem. Soc., 58, 2577-2584 (1936).

18. M. Miller-Andersson, H. Borg, and L.-O. Andersson, "Purification of ATIII by affinity chromatography," Thromb. Res., 5, 439-452 (1974).

19. C. J. v. Delden, G. H. M. Engbers, and J. Feijen, “Interaction of antithrombin III with surface-immobilized albumin-heparin conjugates," J. Biomed. Mater. Res., 29, 1317-1329 (1995).

20. E. Wellisch, "Natural polymers as film formers. Properties of regenerated cellulose," in The Science and Technology of Polymer Films, O. J. Sweeting (ed.), Interscience Publishers, New York, 1968, pp. 139-172.

21. P. K. Smith, A. K. Mallia, and G. T. Hermanson, "Colorimetric method for the assay of heparin content in immobilized heparin preparations," Anal. Biochem., 109, 466-473 (1980).

22. W. L. J. Hinrichs, H. W. M. ten Hoopen, G. H. M. Engbers, and J. Feijen, "Controlled release of heparin by the hydrolysis of covalent bonds," J. Cont. Rel., 45, 163-176 (1997).

23. W. L. J. Hinrichs, H. W. M. ten Hoopen, G. H. M. Engbers, and J. Feijen, "Heparinization of Cuprophan hemodialysis membranes by means of a $N, N^{\prime}$ carbonyldiimidazol activation procedure," to appear.

24. W. L. Chandler, D. D. Solomon, C. B. Hu, and G. Schmer, "Estimation of surface-bound heparin activity: A comparison of methods," J. Biomed. Mater. Res., 22, 497-508 (1988).

25. F. D. Snell, C. T. Snell, and C. A. Snell, "Hexoses and heptoses," in Colorimetric Methods of Analysis, D. Van Nostrand Company, New York, 1961, pp. 181-219.

26. K. A. Smith, C. K. Colton, E. W. Merrill, and L. B. Evans, "Convective transport in a batch dialyzer: determination of true membrane permeability from a single measurement," Chem. Eng. Prog. Symp. Series 84, 64, 45-58 (1968).

27. F. F. Holland, H. E. Gidden, R. G. Mason, and E. Klein, "Thrombogenicity of heparin-bound DEAE cellulose hemodialysis membranes," ASAIO J., 1, 24-36 (1978).

28. J. C. Bokros, V. L. Gott, L. D. L. Grange, A. M. Fadall, K. D. Vos, and M. D. Ramos, "Correlations between blood compatibility and heparin adsorptivity for an impermeable isotropic pyrolytic carbon," J. Biomed. Mater. Res., 3, 497-528 (1969).

29. A. P. Broek, "Characterization of hemodialysis membranes: Membrane structure and function," Thesis, University of Twente, Enschede, The Netherlands, 1993.

30. C. D. Ebert and S. W. Kim, "Immobilized heparin: Spacer arm effects on biological interactions," Thromb. Res., 26, 43-57 (1982).

31. F. Maillet, D. Labarre, and M. D. Kazatchkine, "The role of naturally-occurring antibodies against manmade materials in biocompatibility," Transfus. Sci., 11, 33-41 (1990).

32. A. v. Berlo and D. J. Ellens, "Biocompatibility of haemodialysis membranes," Adv. Exp. Med. Biol., 238, 341358 (1988).

33. J. Yu, N. M. K. Lamba, J. M. Courtney, T. L. Whateley, J. D. S. Gaylor, G. D. O. Lowe, K. Ishihara, and N. Nakabayashi, "Polymeric biomaterials: Influence of phosphorylcholine polar groups on protein adsorption and complement activation," Int. J. Artif. Organs, 17, 499504 (1994).
34. P. Ivanovich, D. E. Chenoweth, R. Schmidt, H. Klinkmann, L. A. Boxer, H. S. Jacob, and D. E. Hammerschmidt, "Symptoms and activation of granulocytes and complement with two dialysis membranes," Kidney Int., 24, 758-763 (1983).

35. M. Diamantoglou, H. D. Lemke, and J. Vienken, "Cellulose-ester as membrane materials for hemodialysis," Int. J. Artif. Organs, 17, 385-391 (1994).

36. A. Mahiout, H. Meinhold, M. Kessel, H. Schulze, and U. Baurmeister, "Dialyzer membranes: effect of surface area and chemical modification of cellulose on complement and platelet activation," Artif. Organs, 11, 149-154 (1987).

37. M. D. Kazatchkine, D. T. Fearon, J. E. Silbert, and F. K. Austen, "Surface-associated heparin inhibits zymosaninduced complement pathway by augmenting the regulatory action of the control proteins on particlebound C3b," J. Exp. Med., 150, 1202-1215 (1979).

38. T. E. Mollnes, V. Videm, J. Riesenfeld, P. Garred, J. L. Svennevig, E. Fosse, K. Hogasen, and M. Harboe, "Complement activation and bioincompatibility. The terminal complement complex for evaluation and surface modification with heparin for improvement of biomaterials," Clin. Exp. Immunol., 86, 21-26 (1991).

39. R. Deppisch, V. Schmitt, J. Bommer, and G. M. Hansch, "Fluid phase generation of terminal complement complex as a novel index of bioincompatibility," Kidney Int., 37, 696-706 (1990).

40. J. P. Black, C. H. Gemmell, E. L. Yeo, and M. V. Sefton, "Assessment of material induced complement activation," Trans. Soc. Biomater., 18, 210 (1995).

41. A. F. Cheung, C. J. Parker, J. Janatova, and E. Brynda, "Modulation of complement activation on hemodialysis membranes by immobilized heparin," J. Am. Soc. Nephrol., 2, 1328-1337 (1992).

42. B. Montdargent, J. Toufik, M. P. Carreno, D. Labarre, and M. Jozefowicz, "Complement activation and adsorption of protein fragments by functionalized polymer surfaces in human serum," Biomaterials, 13, 571576 (1992)

43. D. L. Levett, C. Woffindin, A. G. Bird, N. A. Hoenich, M. K. Ward, and D. N. S. Kerr, "Haemodialysisinduced activation of complement. Effects of different membranes," Blood Purification, 4, 185-193 (1986).

44. V. V. Sinitsyn, E. T. Bokchubaev, A. G. Mamontova, N. V. Ovtrakht, E. L. Nasonov, G. A. Konovalov, and V. V. Kukharchuk, "C3a and C5a anaphylatoxins bind to heparin-based sorbent in low density lipoprotein apheresis: In vitro and in vivo investigations," Artif. Organs, 16, 291-313 (1992).

45. M. Pekna, R. Larsson, B. Formgren, U. R. Nilsson, and B. Nilsson, "Complement activation by polymethylmethacrylate minimized by end-point heparin attachment," Biomaterials, 14, 189-192 (1993).

46. E. Klein, F. F. Holland, and K. Eberle, "Comparison of experimental and calculated permeability and rejection coefficients for hemodialysis membranes," J. Membr. Sci., 5, 173-188 (1979).

47. E. Klein, F. Holland, A. Lebeouf, A. Donnaud, and J. K. Smith, "Transport and mechanical properties of hemodialysis hollow fibers," J. Membr. Sci., 1, 371-396 (1976).

48. H. A. Staab, "New methods of preparative organic chemistry IV. Synthesis using heterocyclic amides (azolides)," Angew. Chem. Int. Ed., 7, 351-367 (1962).

Received January 26, 1996

Accepted May 16, 1996 